\title{
VARIAÇÃO ESPACIAL E TEMPORAL DA BIODISPONIBILIDADE DE METAIS NA REGIÃO ESTUARINA DO COMPLEXO ESTUARINO- LAGUNAR DE IGUAPE-CANANÉIA, SUDESTE DO BRASIL
}

\author{
Júlio César Rodrigues Azevedo* \\ Lilian Dalago Salgado**
}

RESUMO: O Complexo Estuarino-Lagunar de Iguape-Cananéia (CELIC) apresenta condições ambientais distintas entre suas porções Norte e Sul e sofre com diferentes pressões antrópicas. Este estudo avaliou a variação espacial e temporal da biodisponibilidade dos metais $\mathrm{Fe}, \mathrm{Zn}, \mathrm{Mn}, \mathrm{Co}, \mathrm{Cu}, \mathrm{Cr}, \mathrm{Cd}, \mathrm{Pb}$ and $\mathrm{Ni}$, nos sedimentos em 8 pontos do CELIC em 2 períodos de 2013. A avaliação da biodisponibilidade dos metais deu-se através do método da extração simultânea de íons metálicos e sulfetos volatizados por acidificação (SEM/SVA). Foi vista ampla variabilidade espacial e temporal nos parâmetros analisados, onde a média dos metais fortemente biodisponíveis deu-se na ordem de $\mathrm{Fe}>\mathrm{Mn}>\mathrm{Zn}>\mathrm{Pb}>\mathrm{Cu}$. Todos os pontos apresentaram razão SEM/SVA > 1 para os íons metálicos $\mathrm{Pb}, \mathrm{Cd}$, Ni, Cu e Zn, com maior toxicidade dos sedimentos a Norte, sugerindo a biodisponibilidade desses metais e destacando o potencial destes sedimentos de causar efeitos adversos à biota local. Estes resultados salientam as influências do aporte de resíduos urbanos, agrícolas e de atividades mineradoras pretéritas ao sistema, advindos especialmente do Rio Ribeira de Iguape.

PALAVRAS-CHAVE: Chumbo; Estuário; Mineração; Nutrientes.

\section{SPACE AND TIME VARIATION OF METAL BIO-AVAILABILITY IN THE ESTUARY REGION OF THE IGUAPE-CANANÉIA ESTUARY- LAGOON COMPLEX, SOUTHEASTERN BRAZIL}

ABSTRACT: The Iguape-Cananéia estuary-lagoon complex (CELIC) has different environment conditions between the northern and Southern sections, with different anthropic pressures. Current study evaluates the space and time variation of bioavailability of metals $\mathrm{Fe}, \mathrm{Zn}, \mathrm{Mn}, \mathrm{Co}, \mathrm{Cu}, \mathrm{Cr}, \mathrm{Cd}, \mathrm{Pb}$ and $\mathrm{Ni}$ in sediments at 8 sites of CELIC during two periods in 2013. Metal bio-availability was assessed by the method

\footnotetext{
"Pós-Doutor em Ciências Ambientais/Química Ambiental. Docente titular do programa de Pós-Graduação em Ciência e Tecnologia Ambiental da Universidade Tecnológica Federal do Paraná (UTFPR), Brasil.

** Doutora em Ecologia e Conservação pela Universidade Federal do Paraná (UFPR), Brasil.

E-mail: : lilian.salgado@hotmail.com
} 
of simultaneous extractions of metal ions and sulfates volatized by acidification (SEM/ SVA). There was a broad space and time variability in the parameters under analysis where mean availability of metals followed the sequence $\mathrm{Fe}>\mathrm{Mn}>\mathrm{Zn}>\mathrm{Pb}>\mathrm{Cu}$. All sites provided ratio SEM/SVA $>1$ for metal ions $\mathrm{Pb}, \mathrm{Cd}, \mathrm{Ni}, \mathrm{Cu}$ and $\mathrm{Zn}$, with greater toxicity in the northern sediments. The above suggests bio-availability of the metals and highlighted the potential of the sediments, adversely affecting local biota. Results underscore the influence of numerous urban, agricultural and mining activities on the system, mainly derived from the river Ribeira de Iguape.

KEY WORDS: Lead; Estuary; Mining; Nutrients.

\section{INTRODUÇÃO}

Estuários são ambientes de transição entre os sistemas aquáticos continentais e oceânicos. Sofrem grande influência das marés, e apresentam gradientes ambientais que vão desde águas doces, próximo da cabeceira dos rios até águas marinhas, próximo das desembocaduras (BARCELLOS et al., 2005). Oferecem inúmeros serviços ao ecossistema, entre eles o suprimento de água potável e de recursos pesqueiros. São zonas de intensa ocupação humana, sendo utilizados também para a diluição e o descarte de resíduos, acarretando na contaminação destes ecossistemas aquáticos levando a sua degradação (BARBIERI et al., 2014; MACHADO et al., 2016).

Os metais são poluentes de grande interesse ecológico, sendo que dentre eles encontram-se elementos essenciais e não-essenciais para os seres vivos, no entanto em ambos os casos são potencialmente prejudiciais quando em elevados níveis de exposição e absorção. Apresentam propriedades de bioacumulação e biomagnificação e podem persistir durante longos períodos no ambiente (COTTA; REZENDE; PIOVANI, 2006; BORAN; ALTINOK, 2010). Quando de ocorrência natural apresentam concentrações variáveis de acordo com a matriz geológica em que se encontram. Contudo, as atividades antrópicas alteraram sua distribuição, sendo as atividades mineradoras, agrícolas e industriais dentre as principais causas dos aumentos de metais nos ambientes aquáticos (MACHADO et al., 2016).

Os sedimentos funcionam como um importante compartimento nos ecossistemas aquáticos, sendo formados pela deposição de partículas em suspensão 
provenientes de rios, lagos, estuários e oceanos (POMPÊO et al., 2013). Possuem elevadas capacidades de sorção e acumulação, desempenhando um importante papel no destino final de íons metálicos. No entanto, a presença de metais nos sedimentos é complexa pelo fato destes contaminantes poderem existir de diversas formas, variando de acordo com a natureza das fontes que originou o sedimento, transporte e sua deposição no meio (POMPÊO et al., 2013). Assim, a granulometria e a composição dos sedimentos são importantes para a compreensão da hidrodinâmica dos ambientes aquáticos e da dinâmica dos metais neste compartimento (COTTA; REZENDE; PIOVANI, 2006; OLIVEIRA; MARINS, 2011).

A troca de íons entre a coluna da água e o sedimento aumenta na fração granulométrica fina, principalmente pela existência de maior área superficial, mas também por apresentar argilominerais de diferentes grupos, alguns com elevada capacidade de troca de cátions. Assim, as frações mais finas, como silte e argila (<63 $\mu \mathrm{m})$ apresentam maiores concentrações de metais que as frações granulométricas maiores (como a areia). Nesse sentido, baías e estuários podem intensificar a fixação e acumulação de metais nos sedimentos, principalmente nas áreas de manguezais que apresentam abundância de matéria orgânica e frações de argila e silte (AMORIM et al., 2008; SILVEIRA et al., 2013).

Contudo, diversos processos bióticos e abióticos podem remobilizar os metais do sedimento, afetando a qualidade das águas e biodisponibilizando estes elementos, o que permite a bioacumulação e a transferência através da cadeia trófica (BORAN; ALTINOK, 2010). A análise da concentração dos sulfetos volatizados por acidificação (SVA) e biodisponibilidade de íons metálicos extraídos simultaneamente (SEM) tem sido utilizada para averiguar a biodisponibilidade e toxicidade de metais bivalentes nos sedimentos (DI TORO et al., 1992; HOOP et al., 1997; OLIVEIRA et al., 2006; POMPÊO et al., 2013; GARCIA et al., 2014).

Os SVA são encontrados nas águas intersticiais de sedimentos anóxicos e consistem-se primariamente de FeS. Metais bivalentes ( $\mathrm{Cd}, \mathrm{Cu}, \mathrm{Pb}$, Ni e $\mathrm{Zn}$ ) dissolvidos nestas águas podem deslocar o ferro do sulfeto de ferro II (FeS) e originar o sulfeto metálico insolúvel de maior estabilidade, diminuindo sua biodisponibilidade aos organismos aquáticos (DI TORO et al., 1992; HOOP et al., 1997; OLIVEIRA et al., 2006; POMPÊO et al., 2013; GARCIA et al., 2014). Assim, 
a análise do SVA é uma medida que descreve o controle da distribuição de metais nestes ambientes e contribui para a definição de uma provável toxicidade dos sedimentos. Se a quantidade de AVS for maior que o SEM, os metais no sedimento são considerados indisponíveis e os sedimentos, em termos de metais, considerados não tóxicos (SILVEIRA et al., 2013; GARCIA et al., 2014).

O Complexo Estuarino-Lagunar de Iguape-Cananéia (CELIC) está entre as áreas costeiras mais produtivas do Atlântico Sul (MORAIS; ABESSA, 2014). No entanto o sistema passou por diversas mudanças nos últimos séculos, que afetaram a qualidade ambiental da região. A abertura de um canal artificial (Valo Grande), em Iguape no século XIX, desviou cerca de $70 \%$ das águas do rio Ribeira de Iguape para dentro do estuário, fazendo com que o rio se tornasse o maior contribuidor de água doce para o sistema (MAHIQUES et al., 2013). Este rio abrigou diversas minas de $\mathrm{Pb}$, $\mathrm{Zn}, \mathrm{Au}, \mathrm{Ag}$ e As, que operaram durante anos, descartando os rejeitos e as escórias do forno de fundição indiscriminadamente no rio até 1995, contaminando a água e os sedimentos ao longo do seu curso (CASTRO, 2012; MAHIQUES et al., 2013). Estimase que durante o período de mineração o rio tenha recebido aproximadamente 5,5 toneladas por ano de resíduos ricos em metais (GUIMARÃES; SÍGOLO, 2008a). Após o fechamento das minas, os resíduos foram depositados marginalmente ao rio, em forma de pilhas de rejeito, estando expostos às intempéries do tempo e consequentemente à lixiviação (GUIMARÃES; SíGOLO, 2008a).

Os metais sofreram mobilidade através dos sólidos em suspensão, ocasionando a contaminação dos sedimentos ao longo do curso de drenagem do rio, onde diversos estudos indicaram anomalias nas concentrações de metais neste ambiente aquático, especialmente por Pb (CUNHA et al., 2005; GUIMARÃES; SÍGOLO, 2008a; MAHIQUES et al., 2009; MALUF, 2009; CASTRO, 2012; ABESSA et al., 2012; MAHIQUES et al., 2013). Ao longo dos anos, esta contaminação atingiu o CELIC e apesar das concentrações de $\mathrm{Pb}$ nas águas e nos sedimentos terem diminuído consideravelmente (MAHIQUES et al., 2013; ABESSA et al., 2014), o sistema permanece enfraquecido pela carga de resíduos minerais recebidos. Desta forma, este estudo objetivou avaliar as variações espaciais e temporais dos metais $\mathrm{Fe}$, $\mathrm{Zn}, \mathrm{Mn}, \mathrm{Co}, \mathrm{Cu}, \mathrm{Cr}, \mathrm{Cd}, \mathrm{Pb}$ e Ni e a sua biodisponibilidade nos sedimentos do CELIC. 


\section{MATERIAIS E MÉTODOS}

A amostragem foi realizada no Complexo Estuarino-Lagunar de IguapeCananéia (CELIC), que possui área total de $2.500 \mathrm{~km}^{2}$ e situa-se no extremo Sul do Estado de São Paulo, entre as latitudes de $24^{\circ} 50^{`}$ a $25^{\circ} 10^{`} \mathrm{~S}$ e longitudes $47^{\circ} 25^{`}$ a $48^{\circ} 00 ` W$ (Figura 1). Compreende um complexo sistema de canais, entre quatro grandes ilhas (Cardoso, Cananéia, Comprida e Iguape). Estes são denominados de Mar de Cubatão, Mar de Cananéia e Mar Pequeno, com 6, 10 e 6 metros de profundidade média, respectivamente. O sistema é separado do Oceano Atlântico pela Ilha Comprida (70 km de extensão), tendo como limites Sul e Norte as desembocaduras de Cananéia e Icapara (TESSLER; SOUZA, 1998).

A cobertura vegetal é composta por extensa área de Mata Atlântica com manguezais dominando as margens do sistema, sendo os núcleos históricos de Cananéia e de Iguape os principais centros urbanos (MALUF, 2009).

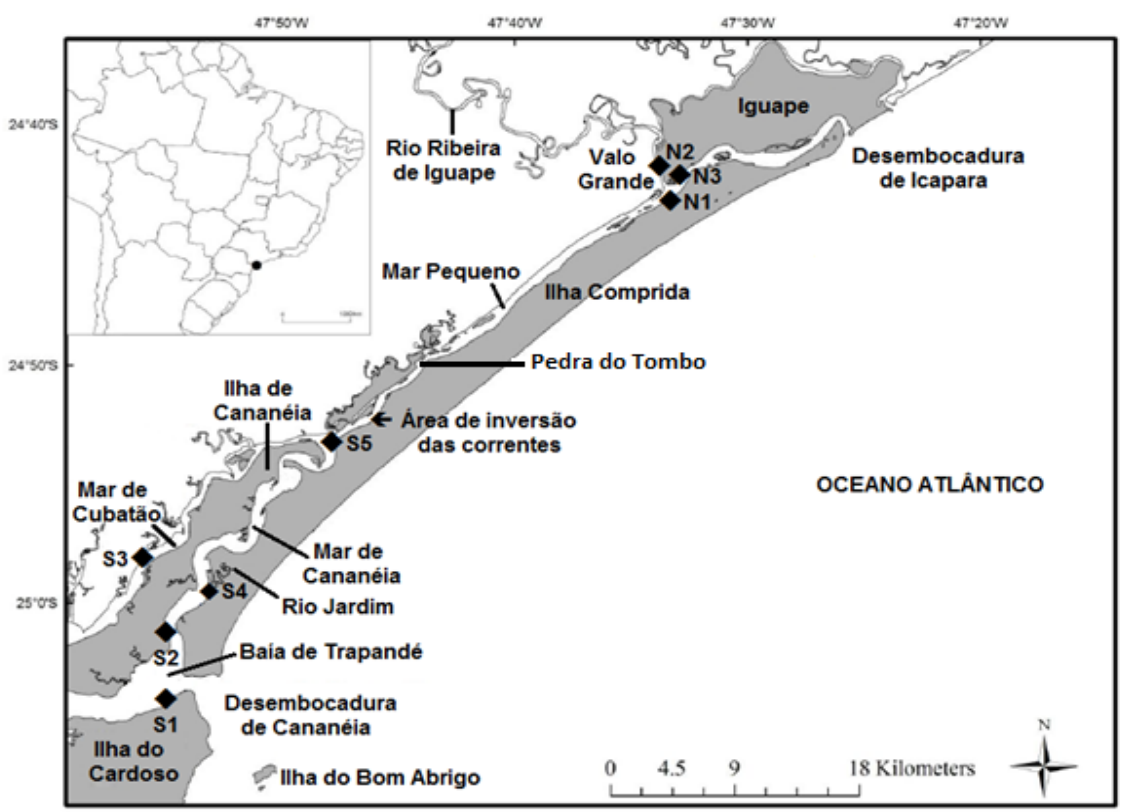

Figura 1. Mapa do Complexo Estuarino-Lagunar de Iguape-Cananéia com destaque para os pontos de coleta. Cinco pontos ao Sul (S1, S2, S3, S4 e S5) e três ao Norte (N1, N2 e N3). 
As coletas foram realizadas em duas diferentes campanhas amostrais: agosto/2013 (C1) e novembro/2013 (C2). O sedimento superficial foi amostrado em 8 pontos ao longo dos canais que ligam Cananéia à Iguape (Figura 1), cinco deles localizados na região Sul (S1 ao S5), próximos ao Sul da Ilha Comprida, e três na porção Norte (N1 ao N3), próximos à cidade de Iguape.

A amostragem foi realizada com o auxílio da draga de Petersen modificada, lançada três vezes, coletando sedimento até uma profundidade de $20 \mathrm{~cm}$, gerandose amostras compostas. Os sedimentos foram homogeneizados e transferidos para sacos de amostragem Whirl Pack, sendo coletados aproximadamente $200 \mathrm{~g}$. As amostras foram acondicionadas em recipientes térmicos para o transporte ao laboratório, no qual as sub-amostras foram congeladas para melhor preservação até a realização das análises. A profundidade foi medida através do sonar Fishfinder Garmin Echo 101 do barco Boto-Cinza II do Instituto de Pesquisas Cananéia (IPeC Cananéia, São Paulo).

Todos os materiais utilizados durante os procedimentos foram previamente descontaminados com solução de ácido nítrico $(10 \%)$ por 24 horas. Foram analisados, em triplicata, os teores de nitrogênio e fósforo nas amostras de sedimento. Estas foram previamente secas em estufa a $60{ }^{\circ} \mathrm{C}$, homogeneizadas e maceradas. O nitrogênio total foi determinado após digestão por persulfato de potássio e o fósforo total por ignição com $\mathrm{HCl}$ (ANDERSEN, 1976). A análise granulométrica foi determinada em $50 \mathrm{~g}$ do sedimento, previamente seco em estufa a $60{ }^{\circ} \mathrm{C}$, homogeneizado e então submetido a peneiramento em mesa agitadora Bertel por 15 min e 3000 Hertz de vibração com peneiras de aberturas 0,$250 ; 0,150 ; 0,106 ; 0,053 ; 0,045$; e $0,038 \mathrm{~mm}$ para a separação das diferentes frações granulométricas (CONAMA, 2004).

A determinação dos sulfetos volatilizados por acidificação (SVA) e biodisponibilidade de íons metálicos extraídos simultaneamente (SEM) foi realizada através de sistema de purga e aprisionamento (purge and trap - Figura 2) (DI TORO et al., 1992). As amostras foram submetidas a um ataque com $10 \mathrm{~mL} \mathrm{de} \mathrm{HCl}$ 6,0 mol $\mathrm{L}^{-1}$ a frio, sob purga de gás de nitrogênio $\left(\mathrm{N}_{2}\right)$ para produzir sulfeto de hidrogênio gasoso $\left(\mathrm{H}_{2} \mathrm{~S}\right)$. Este sulfeto foi arrastado pelo $\mathrm{N}_{2}$, que foi borbulhado e recolhido em uma solução de $150 \mathrm{~mL}$ de $\mathrm{NaOH} 0,1 \mathrm{~mol} \mathrm{~L}^{-1}$.

$\mathrm{O}$ ácido sulfídrico arrastado reage com o $\mathrm{NaOH}$, originando $\mathrm{Na}_{2} \mathrm{~S}$, que permanece em solução. Foi empregado $0,5 \mathrm{~g}$ do sedimento in natura, onde 
as amostras permaneceram por 1 hora no sistema com vazão controlada do gás de arraste. A quantificação do sulfeto foi realizada empregando o método de espectrofotometria pelo azul de metileno (APHA, 2005).

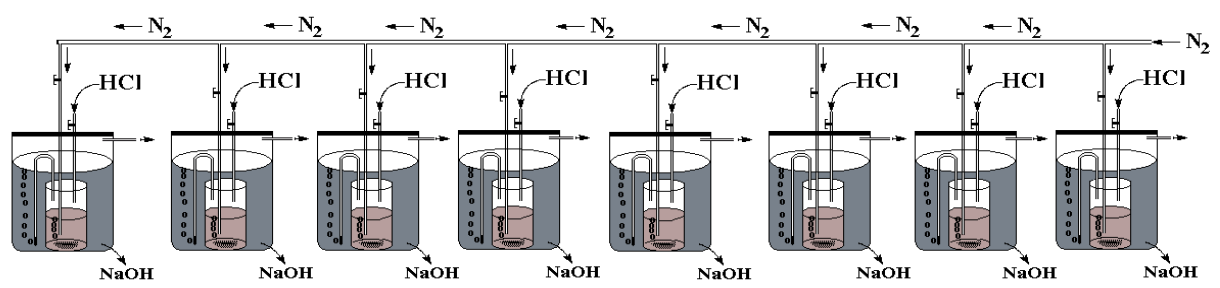

Figura 2. Sistema para a extração, arraste e retenção do sulfeto volatilizado por acidificação.

Para a análise da presença dos metais extraídos com o sulfeto, a solução com material em suspensão (Figura 2), existente no reator foi centrifugada, sedimentando o material em suspensão e posteriormente filtrado em membrana de acetato de celulose $0,45 \cdot \mathrm{m}$. A determinação da concentração dos metais foi realizada através da técnica de espectrometria de absorção atômica com chama (FAAS), no equipamento GBC - Avanta.

A soma das concentrações molares dos metais ( $\mathrm{Cd}, \mathrm{Cu}, \mathrm{Ni}, \mathrm{Pb}$ e $\mathrm{Zn}$ ), na massa seca do sedimento, foram relacionadas com as concentrações molares dos sulfetos para verificar a sua biodisponibilidade. Para isso utilizou-se a razão matemática (equação 1) da soma das concentrações molares dos íons metálicos extraídos simultaneamente (SEM) pela concentração de sulfetos extraídos por ataque ácido a frio (SVA):

\section{$\Sigma[\mathrm{SEM}] /[\mathrm{SVA}]$}

A eficiência de recuperação do sulfeto arrastado e retido em $\mathrm{NaOH}$ foi comparada com padrões preparados em $100 \mathrm{~mL}$ de $\mathrm{NaOH}$. Somente após os ajustes com recuperação acima de $95 \%$ foram iniciadas as análises nos sedimentos. Também foi avaliada a recuperação da adição de padrão de sulfeto nas amostras de sedimento. Sub-amostras $(n=5)$ de sedimentos foram pesadas, liofilizadas e calculadas sua massa seca, convertendo assim a quantidade de SVA e metais para massa seca de sedimento. Para avaliar diferenças ambientais entre pontos localizados na região 
Norte e Sul do estuário, e entre as coletas foi utilizada uma Análise de Variância Multivariada Permutacional - PERMANOVA.

\section{RESULTADOS E DISCUSSÃO}

A granulometria dos sedimentos apresentou 97\% de frações de areias, 2\% de frações de silte e $1 \%$ de frações de argila. A granulometria não variou entre os pontos localizados ao Norte e Sul ( $\mathrm{F}=0,01 ; p=0,872$ ), nem entre os períodos de amostragem $(\mathrm{F}=0,02 ; \mathrm{p}=0,941)$. A predominância de sedimentos arenosos nos pontos amostrados provavelmente deve-se à influência da hidrodinâmica local. Tessler e Souza (1998) também evidenciaram que a maioria dos sedimentos do fundo dos canais do CELIC é arenosa (75\% das amostras). Investigações anteriores mostram que as regióes das desembocaduras de Cananéia e de Icapara e da área de confluência dos canais do Mar Pequeno, Mar de Cubatão e Mar de Cananéia apresentam baixos conteúdos de lamas, em decorrência da forte dinâmica de correntes junto ao fundo a que estas áreas estão submetidas, função da proximidade com as desembocaduras do estuário (TESSLER; SOUZA, 1998; BARCELLOS et al., 2005).

No sedimento, as concentrações de N-Total (Figura 3A) variaram de 34,4 $( \pm 5,6)$ a $77,2( \pm 7,1) \mathrm{mg} \mathrm{kg}^{-1}$, possuindo maior influência nos pontos localizados na porção Sul do estuário, com os maiores valores obtidos na coleta de agosto/13 (C1). Embora tenha sido observada similaridade na composição das frações sedimentares do CELIC, geralmente, valores mais elevados de nitrogênio foram encontrados junto a frações granulométricas formadas por partículas finas. Sedimentos compostos especialmente de silte e argila apresentam maior teor de carbono orgânico, e, consequentemente, maior tendência de adsorção de compostos orgânicos hidrofóbicos (AMORIM; FÁVARO; BRAGA, 2011). Barcellos e Furtado (2005) atribuíram a distribuição do nitrogênio total no sistema em concordância com a distribuição de lama nos sedimentos, com maiores teores associados também às áreas de baixa hidrodinâmica. Os maiores valores reportados no inverno podem ser devido ao maior tempo de residência observado para as águas dentro do estuário neste período, do que no verão (BRICHTA; GAETA, 2000). 

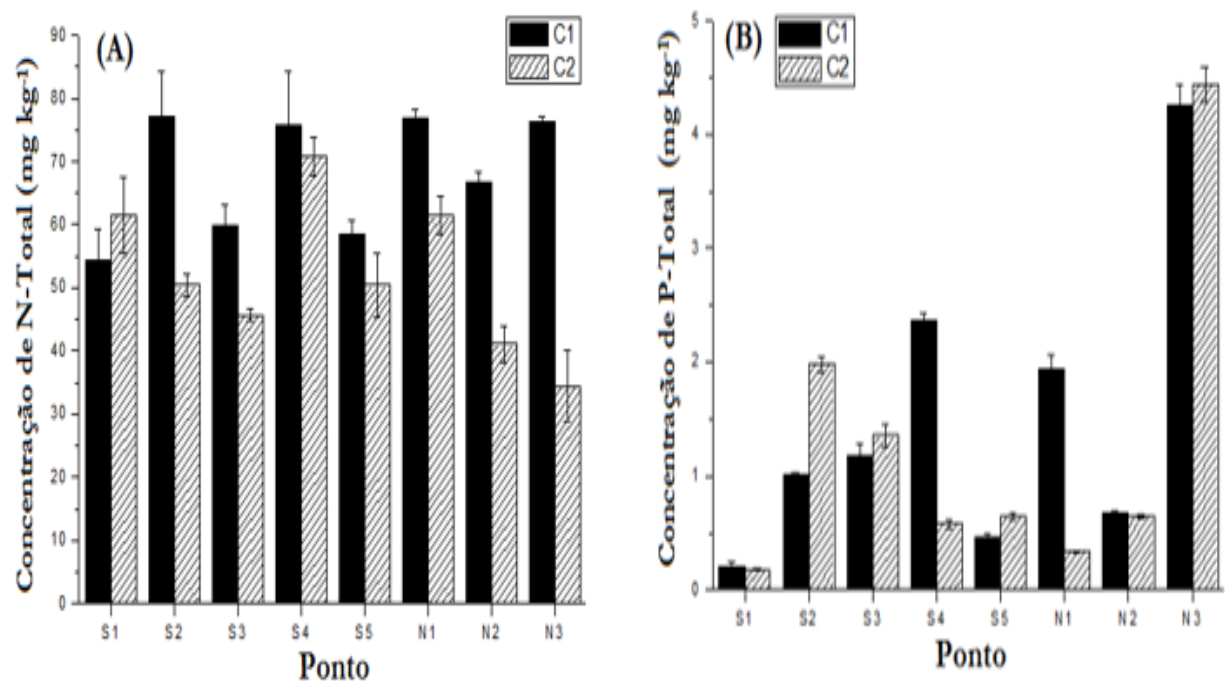

Figura 3. Variação da concentração de nitrogênio total (A) e fósforo total (B) (em mg kg$\left.{ }^{-1}\right)$ nos sedimentos dos pontos amostrais da região Sul (S1, S2, S3, S4 e S5) e região Norte (N1, N2 e N3) nas diferentes coletas (C1: agosto/2013 e C2: novembro/2013).

Em termos de fósforo total (P-Total) as concentrações (Figura 3B) variaram de $0,18( \pm 0,01)$ a $4,44( \pm 0,15) \mathrm{mg} \mathrm{kg}^{-1}\left(0,006\right.$ a $\left.0,143 \mu \mathrm{mol} \mathrm{g}^{-1}\right)$ com menores valores registrados para o ponto S1 e maiores valores para o ponto $\mathrm{N} 3$, independente da coleta. As divergências nos conteúdos de fósforo nos sedimentos da região podem estar relacionadas com as condições ambientais e pressões antrópicas entre as porções Norte e Sul, com as maiores pressões antrópicas vistas em Iguape (ao Norte) com o desague do Rio Ribeira de Iguape e a maior ocupação humana vista na região.

Em Cananéia (Sul) encontram-se características típicas de ambiente estuarino, com grande influência marinha. De maneira geral a descarga de efluentes nesta região foi relativamente menor, em função da menor ocupação humana, sendo os maiores valores de P-Total registrados nos pontos S2 na primavera (C2) e em S4 no inverno (C1). O ponto $\mathrm{S} 2$ fica localizado em frente à cidade de Cananéia, estando sujeito ao lançamento de esgotos domésticos e deposição de lixo (MORAIS; ABESSA, 2014), que tornam o local susceptível a este tipo de contaminação. O ponto S4, localizado na saída do Rio Jardim, pode sofrer influência das fontes terrígenas de fósforo trazidos pelo rio.

Já em Iguape (Norte) encontram-se características predominantemente 
fluviais, devido ao desague do Rio Ribeira de Iguape, o qual carrega para o estuário grande aporte de fósforo de fontes terrígenas e antrópicas. No presente estudo, o ponto N3 apresentou o maior conteúdo de P-Total e está localizado em frente ao mercado de Iguape, próximo à saída deste rio. Este drena vastas áreas de agricultura de banana e chá, que acarretariam em um aporte de fosfatos para o sistema, pela lixiviação dos solos com fertilizantes, recebendo ainda material oriundo das atividades de mineração de fosfato efetuado pela CIA de Mineração (BARCELLOS et al., 2005; MAHIQUES et al., 2009). Adicionalmente, o rio corta o centro urbano de Iguape e recebe parte dos efluentes domésticos, sem tratamento, oriundos da cidade.

Barcellos et al. (2005) observaram concentrações de P-total que variaram entre 1,42 a $30,15 \mu \mathrm{mol} \mathrm{g}^{-1}$ nos sedimentos do mesmo estuário e observaram a correlação deste parâmetro com os sedimentos ricos em conteúdo de pelitos. Ainda de acordo com os autores, as fontes terrígenas de fósforo são trazidas pelos rios e pelo escoamento primário superficial, havendo maior influência de material de origem terrígena ao Norte e maior influência de material de origem marinha no Sul da região.

No geral os resultados indicaram que os sedimentos coletados, provavelmente, foram depositados recentemente, sem longa acumulação no local devido à dinâmica fluvial com alta energia, transportando o material mais fino em suspensão, confirmados pelos baixos teores de silte, argila, P-total e N-total.

O sulfeto volatilizado por acidificação (SVA) teve variação de 0,061 a 14,67 mmol kg-1 entre os pontos amostrais. As maiores médias foram observadas nos pontos da porção Sul do estuário, em S2 na amostragem de inverno (C1), e em S1 na coleta da primavera (C2) (Figura 4). Avaliando os sedimentos do sistema estuarino de Santos-Cubatão, Nizolli e Luiz-Silva (2009) encontraram variação similar de SVA nas camadas superficiais, com valores médios variando de $0,39 \mathrm{mmolkg}^{-1}$ no verão a 2,12 mmolkg-1 no inverno, sendo estes valores inferiores aos encontrados em camadas mais profundas do sedimento. Baixos valores de SVA nas camadas mais superficiais dos sedimentos podem ser atribuídos à exposição destes sedimentos à bioturbação e o fato da água ser óxica, provocando a oxidação de sulfetos a sulfatos, diminuindo os níveis de SVA, sendo este comportamento observado em estuários (CAMPANA; RODRÍGUEZ; BLASCO, 2004). 
Outro aspecto que causa influência sobre os resultados é o fato de o sedimento ser predominantemente arenoso, com menor quantidade de matéria orgânica, ou seja, com menor dinâmica biológica no sistema.

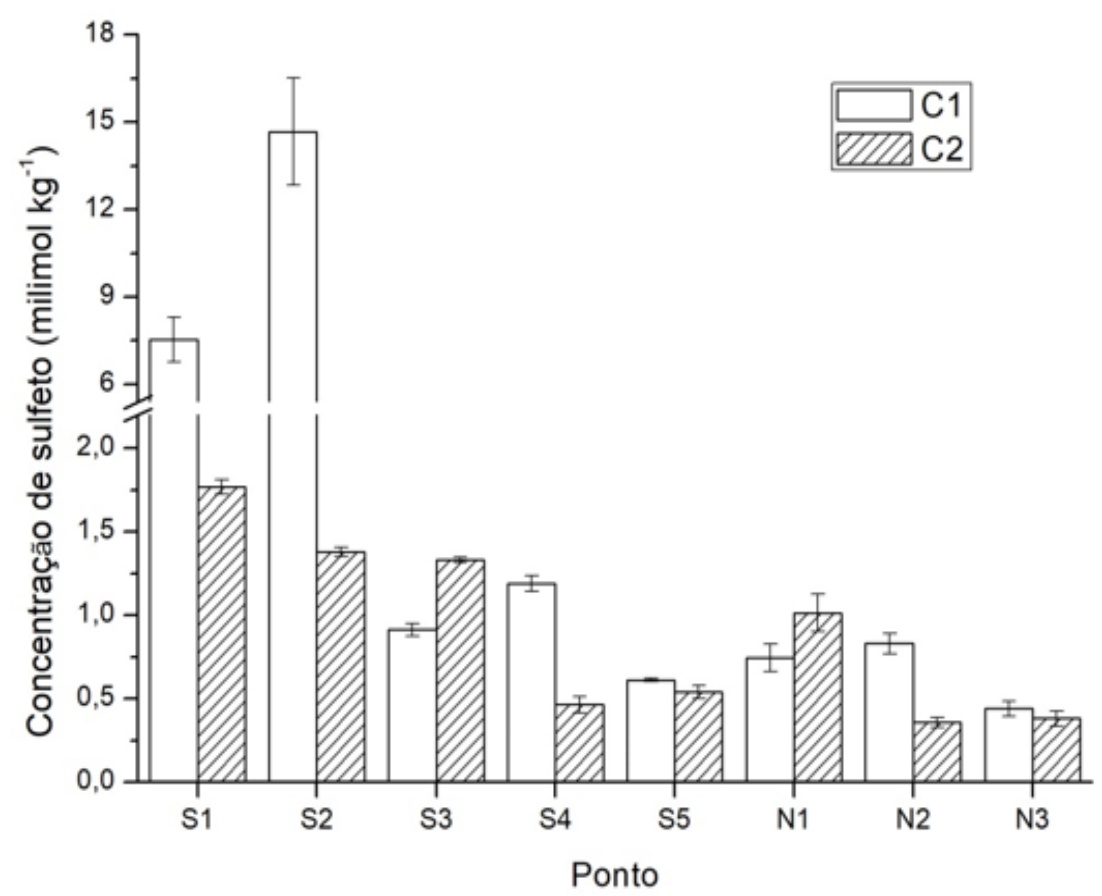

Figura 4. Variação da concentração de sulfeto volatilizado por acidificação (SVA) nos sedimentos, com desvio padrão $(\mathrm{N}=3)$, em mmol SVA por kg de sedimento, nos pontos amostrais da região Sul (S1, S2, S3, S4 e S5) e região Norte (N1, N2 e N3) entre as diferentes coletas (C1: agosto/2013 e C2: novembro/2013).

Em termos de metais fortemente biodisponíveis, as maiores concentrações encontradas foram dos íons de ferro (Figura 5A) e manganês (Figura 5B), que variaram de 4.740 a $22.007 \mathrm{mg} \mathrm{kg}^{-1}$ e de 5,7 a $7776 \mathrm{mg} \mathrm{kg}^{-1}$, respectivamente, podendo em sua maior parte prover de fontes pedogênicas. Apesar das elevadas concentrações observadas por todo o sistema, na região Sul, no ponto S3, foram obtidas as menores concentrações. Já os valores superiores de Fe e Mn foram observados nos pontos N1 e N3, região Norte, em ambos os períodos (Figura 5A e 5B). 

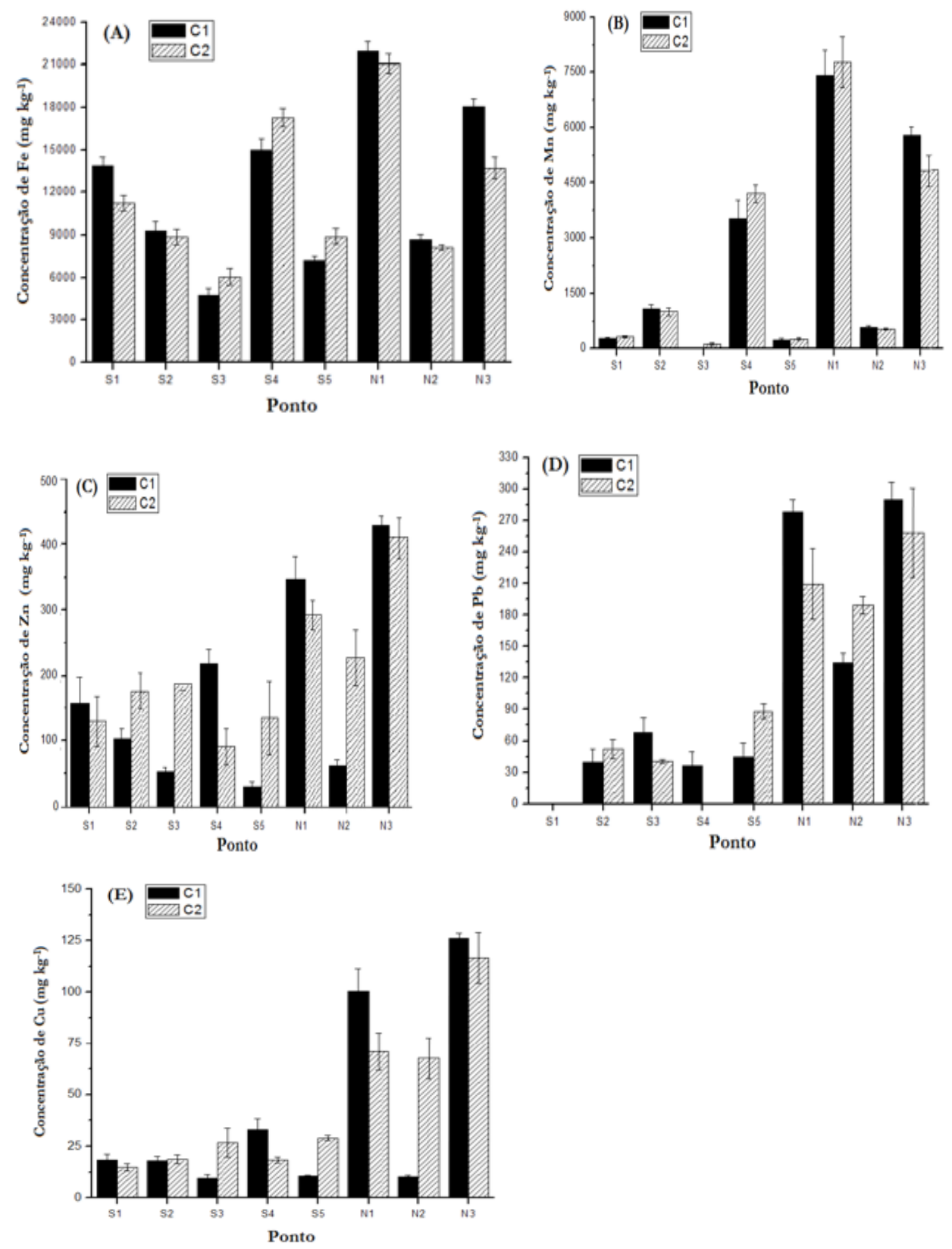

Figura 5. Variação das concentrações dos íons ferro (A), manganês (B), zinco (C), chumbo (D) e cobre (E) nos sedimentos dos pontos amostrados na região Sul (S1, S2, S3, S4 e S5) e região Norte (N1, N2 e N3) nas coletas C1 (agosto/2013) e C2 (novembro/2013). 
Para o íon zinco (Figura 5C), a variação foi de 29,8 a $429,9 \mathrm{mg} \mathrm{kg}^{-1}$. As maiores concentrações do Zn também foram vistas nos pontos N1 e N3, nas duas coletas. Já as concentrações de chumbo (Figura 5D) variaram de abaixo do <LD a 290,3 mg $\mathrm{kg}^{-1}$, sendo que em ambas as amostragens no ponto $\mathrm{S} 1$, os valores não excederam $\mathrm{o}$ LD. Já as maiores concentrações de $\mathrm{Pb}$ foram também nos pontos da região Norte (N1, N2 e N3). Para o íon cobre, a variação das concentrações foi de 9,57 a 127,3 mg $\mathrm{kg}^{-1}$ (Figura 5E), com menores valores encontrados no Sul (ponto S3, no inverno (C1)) e as maiores concentrações de cobre foram encontradas nos pontos ao Norte (N1, N2 e N3). Os íons metálicos de Cd, Co, Cr e Ni apresentaram concentrações abaixo do LD e trações em alguns casos.

Comparando-se as concentrações dos metais biodisponíveis observados nos sedimentos do CELIC com a legislação vigente do órgão ambiental brasileiro, Resolução CONAMA n ${ }^{0} 344$ (2004), foi possível observar que as concentrações de $\mathrm{Zn}, \mathrm{Cu}$ e $\mathrm{Pb}$ excedem os limites estabelecidos em alguns dos pontos avaliados. Segundo a legislação, os valores de nível I indicam o limiar abaixo do qual prevêse baixa probabilidade de efeitos adversos à biota e o de nível II o limiar acima do qual prevê-se um provável efeito adverso à biota (CONAMA, 2004). Para o Zn as concentrações excederam o limite de nível I (150 mg kg-1), nos pontos S1, S4 e N1 na coleta $\mathrm{C} 1$ e nos pontos S2, S3, N1 e N2 na coleta C2; e o limite de nível II (410 $\mathrm{mg} \mathrm{kg}^{-1}$ ) no ponto N3 em ambas as coletas. Para o $\mathrm{Cu}$ os pontos N1 e N3 na coleta C1 e os pontos N1, N2 e N3 na coleta C2 excederam o limite de nível I (34 mg kg ${ }^{-1}$ ), enquanto nenhum excedeu o valor de $270 \mathrm{mg} \mathrm{kg}^{-1}$, limite de nível II. Para o chumbo, o limite de nível I, de $46,7 \mathrm{mg} \mathrm{kg}^{-1}$, foi excedido nos pontos S3 e N2 na coleta C1 e nos pontos S2, S5, N1 e N2 na coleta C2. Já o valor de $218 \mathrm{mg} \mathrm{kg}^{-1}$, limite de nível II, foi excedido no ponto $\mathrm{N} 1$ na coleta $\mathrm{C} 1$ e no ponto $\mathrm{N} 3$ nas duas coletas.

Estes resultados demonstram que somadas à geologia local, as atividades antrópicas podem interferir na distribuição dos metais nos sedimentos do CELIC. Para todos os metais estudados os pontos de menores concentrações foram registrados ao Sul do sistema. Esta região encontrou-se submetida a um menor impacto antrópico, devido à menor ocupação humana e a ausência de atividades industriais expressivas (MALUF, 2009; AMORIM; FÁVARO; BRAGA, 2011). No entanto, existe a insuficiência da rede de esgotos nas cidades da região, o que faz com que o material seja despejado in natura nos rios ou diretamente no mar (MALUF, 2009; 
BARBIERI et al., 2014; MORAIS; ABESSA, 2014). Assim, o lançamento de esgotos domésticos, as atividades agrícolas de entorno e a presença de embarcações ainda constituem fontes potenciais de poluição por metais a esta região.

Contudo, os impactos das ações antrópicas no sistema foram mais evidentes nas proximidades de Iguape, devido à existência do maior centro urbano, a presença de algumas poucas atividades industriais e, em especial, ao histórico de mineração na região (GUIMARÃES; SÍGOLO, 2008b; MAHIQUES et al., 2013). Além disso, a economia do Vale do Ribeira baseia-se, em grande parte, da agricultura do chá, arroz e banana que demandam do uso de fertilizantes nas plantações, que podem estar contribuindo com o aporte de metais ao CELIC, via Valo Grande (MALUF, 2009; CASTRO, 2012).

Foram observadas algumas correlações positivas entre os metais. A concentração do íon cobre foi correlacionada com a dos íons zinco $(\mathrm{R}=0,9549 ; \mathrm{p}$ $<0,001)$, chumbo $(\mathrm{R}=0,9148 ; \mathrm{p}<0,001)$, manganês $(\mathrm{R}=0,7527 ; \mathrm{p}=0,001)$, e ferro $(\mathrm{R}=0,6130 ; \mathrm{p}<0,012)$. A concentração do zinco foi correlacionada positivamente com as de chumbo $(\mathrm{R}=0,8123 ; \mathrm{p}<0,001)$, manganês $(\mathrm{R}=0,7457$; $\mathrm{p}=0,001)$, e ferro $(\mathrm{R}=0,6465 ; \mathrm{p}=0,007)$. A concentração de chumbo, além de correlacionar com a do cobre e zinco, também correlacionou com a concentração do manganês $(\mathrm{R}=0,6872 ; \mathrm{p}=0,003)$ e não apresentou correlação significativa com a concentração de ferro $(\mathrm{R}=0,4913 ; \mathrm{p}=0,053)$. Estas correlações indicam que, provavelmente, os metais $\mathrm{Zn}$, $\mathrm{Cu}$ e $\mathrm{Pb}$ sejam provenientes de mesma fonte, ou seja, das antigas atividades de mineração das áreas próximas e que a entrada destes metais ao sistema ainda persiste, confirmando a contribuição das pilhas de rejeitos existentes nas margens do Rio Ribeira de Iguape.

Os pontos próximos à cidade de Iguape (N1, N2 e N3) se mostraram afetados em maior grau pelas concentrações de metais, corroborando com estudos feitos anteriormente no sistema. Para a região Sul, Amorim, Fávaro e Braga (2011) encontraram nos sedimentos estuarinos próximos à Cananéia valores abaixo dos limites TEL (Threshold Effect Level) e PEL (Probable Effect Level) para os metais tóxicos $\mathrm{Cd}, \mathrm{Pb}$ e $\mathrm{Hg}$, demonstrando que não haviam grandes influências antrópicas na região Sul do estuário.

Para a região Norte, valores de $\mathrm{Pb}$ acima dos níveis estabelecidos na literatura foram encontrados nos sedimentos por Saito et al. (2001) e por Guimarães e Sígolo 
(2008b), que relataram valores acima do TEL, para o mesmo metal nos sedimentos próximos a Iguape. Mahiques et al. (2009) observaram que os valores de $\mathrm{Pb}, \mathrm{Cu}$ e Cr nestes sedimentos eram duas vezes maiores do que os encontrados nos sedimentos contaminados do estuário de Santos, uma das áreas mais industrializadas da zona costeira brasileira.

A escória de forno e o rejeito produzido durante os processos metalúrgicos foram lançados diretamente no Rio Ribeira de Iguape e desde a abertura do Valo Grande, parte desta contaminação atinge o CELIC. Após o encerramento da mineração (meados de 1990) os resíduos da mineração foram acumulados marginalmente às drenagens do rio, na forma de imensas pilhas de rejeito, que subsistem até hoje, expostas às intempéries, em especial chuvas e ventos (CUNHA et al., 2005; MAHIQUES et al., 2009; RODRIGUES et al., 2012; CASTRO, 2012).

Guimarães e Sígolo (2008b) avaliaram a composição dos resíduos da metalurgia na região e revelaram que os mesmos possuem teores extremamente elevados de $\mathrm{Zn} \mathrm{e} \mathrm{Pb}$, apresentando também composição por $\mathrm{Cu}, \mathrm{Cr}$ e $\mathrm{Ba}$, estes metais sofrem incorporação aos materiais em suspensão e podem ser transportados, ao longo do curso do rio, até a região estuarina, próxima a Iguape. Segundo Abessa et al. (2012) isto se deve a alta energia hidrodinâmica da região do Alto e Médio Rio Ribeira de Iguape, e que devido à declividade, o esperado é que não ocorra deposição acentuada de material fino contendo metais nestas áreas, de modo que o material contaminado tende a ser levado para as regióes mais baixas do rio, no caso o CELIC.

Os íons metálicos de $\mathrm{Co}, \mathrm{Cd}, \mathrm{Cr}$ e Ni praticamente não foram detectados neste estudo, sendo obtidas somente concentrações-traço nos pontos N1 e N3 de cromo, cádmio e cobalto. Estes resultados estão de acordo com a literatura, que não reportam altas concentrações de Cd para os sedimentos e águas da região (ALBA; SOUZA FILHO; FIGUEIREDO, 2008; MALUF, 2009). Embora o Rio Ribeira de Iguape tenha recebido material rico em cromo durante as atividades mineradoras (CUNHA et al., 2005), altas concentrações do metal não são observadas nos sedimentos da região Norte do CELIC.

Com os passar dos anos as concentrações dos metais nos sedimentos caíram consideravelmente, provavelmente, pelo fato do ambiente estar passando por processos de restauração naturais (MAHIQUES et al., 2013; ABESSA et al., 
2014). Armelin e Saiki (2009) realizaram a caracterização multielementar em um testemunho de sedimentos (core) coletado no Valo Grande e observaram uma queda abrupta nas concentrações de Pb. Mahiques et al. (2009), através da análise de amostras estratificadas do sedimento local, relataram que durante as atividades mineradoras o ambiente apresentava significativa poluição por $\mathrm{Pb}$, sendo observado que a contaminação nos sedimentos da região vem diminuindo após a paralisação destas atividades. No entanto, foi observado nesta pesquisa que, ainda hoje, nas proximidades de Iguape, os sedimentos ainda apresentam concentrações preocupantes de alguns metais, especialmente do chumbo, os quais, pelo método de extração, são considerados como fortemente biodisponíveis.

Todos os pontos amostrados no CELIC apresentaram a razão [SEM/SVA] $>1$, ou seja, apresentam sedimentos potencialmente tóxicos, em maior ou menor grau. Esta toxicidade pode ocorrer pela existência de pouco sulfeto nos sedimentos em relação à maior quantidade encontrada de metais bivalentes (HOOP et al., 1997), como visto nos sedimentos estudados (Figura 6).

Apesar de se observar um decréscimo da biodisponibilidade dos metais nos sedimentos em direção à Cananéia (Sul), todos os pontos também apresentaram razão [SEM/SVA] > 1 em uma das coletas, sendo considerados como potencialmente tóxicos, podendo também, sob certas condições, trazer efeitos à biota.

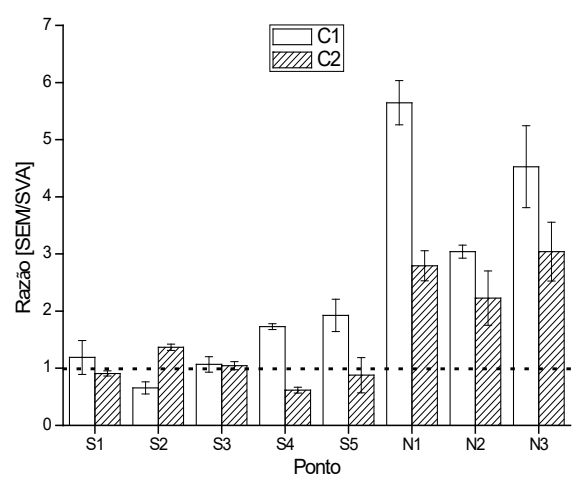

Figura 6. Variação da razão [SEM/SVA] determinadas nas amostras de sedimentos da região Sul (S1, S2, S3, S4 e S5) e região Norte (N1, N2 e N3) do Sistema Estuarino-Lagunar de Iguape-Cananéia nas coletas (C1: Inverno e C2: Primavera). 
Os ambientes com maior toxicidade em relação às concentrações de metais foram os pontos amostrados ao Norte (N1, N2 e N3), próximos a cidade de Iguape. Guimarães e Sígolo (2008b) observaram altos teores de metais (Zn, Cu e Pb) próximo a este centro urbano e afirmam que os baixos valores de $\mathrm{pH}$ e do potencial redox obtidos pela pesquisa, possivelmente, estejam contribuindo para liberação desses metais (mobilidade). Este fato pode ser a causa da aumentada biodisponibilidade na região, o que consequentemente aumentam as probabilidades destes elementos serem retidos pelos organismos aquáticos e participarem da cadeia alimentar (GUIMARÃES; SÍGOLO, 2008; BORAN; ALTINOK, 2010; GUSSO-CHOUERI et al., 2018).

Ainda que o Fe, $\mathrm{Zn}$, Mn e $\mathrm{Cu}$ sejam metais essenciais e participem de diversos processos biológicos, em altas concentrações tornam-se tóxicos, da mesma forma que os metais não essenciais. Neste sentido, ganham atenção especial os teores de $\mathrm{Pb}$ nos sedimentos, uma vez que não possui função biológica conhecida aos organismos, sendo altamente tóxico, mesmo em baixas concentrações.

A maior parte do chumbo é retida nos sedimentos e pequena parte pode ser transportada em águas de superfície ou subterrâneas. Contudo, mudanças nas condições ambientais podem afetar sua biodisponibilidade, fazendo com que sejam novamente disponibilizados para a coluna d'água, graças a reações de oxi-redução, ou a processos de ressuspensão de origem física (correnteza), biológica (atividade dos organismos que vivem nos sedimentos) e humana (dragagem e navegação) (COTTA; REZENDE; PIOVANI, 2006).

Estes processos podem fazer com que estes elementos fiquem disponíveis para a biota (MALUF, 2009; BORAN; ALTINOK, 2010). Investigações com a biota local revelam a alta biodisponibilidade de metais em ambas as regiões, por meio da incorporação destes elementos por diferentes espécies de moluscos (MACHADO et al., 2002; GUIMARÃES; SÍGOLO, 2008a) e peixes (CASTRO, 2012; FERNANDEZ et al., 2014; GUSSO-CHOUERI et al., 2018).

Para a região do Rio Ribeira de Iguape, estudos com a avaliação de chumbo em bivalves feitos por Guimarães e Sígolo (2008a), com a espécie Corbicula flumínea, e por Rodrigues et al. (2012) com Anodontites tenebricosus, encontraram em média as concentrações de $2,41 \mathrm{mg} \mathrm{kg}^{-1} \mathrm{e} 4,40 \mathrm{mg} \mathrm{kg}^{-1} \mathrm{de} \mathrm{Pb}$, respectivamente. 
Em ambos os estudos, as concentrações nos moluscos foram comparáveis às regiões moderadamente contaminadas, sendo que em A. tenebricosus os valores foram superiores aos 3,0 $\mathrm{mg} \mathrm{kg}^{-1}$ permitidos para o consumo humano no Brasil (ANVISA, 1998). Castro (2012), analisando a biodisponibilidade do Pb na bacia hidrográfica do Rio Ribeira do Iguape e seus afluentes por meio dos teores dos sedimentos e do sangue de peixes, observou uma forte correlação entre as concentrações do metal nas duas matrizes amostrais. O autor obteve no ponto de maior contaminação o valor de $474 \mathrm{mg} \mathrm{kg}^{-1}$ de $\mathrm{Pb}$ nos sedimentos e $118 \mu \mathrm{g} \mathrm{L}-1$ no sangue dos peixes, concluindo que a região se encontrava altamente contaminada (CASTRO, 2012).

Para a região estuarina do CELIC, na avaliação de metais essenciais Machado et al. (2002) observaram concentrações de $\mathrm{Zn}$ acima do limite de $50,0 \mathrm{mg} \mathrm{kg}^{-1} \mathrm{em}$ ostras (Crassostrea brasiliana) da região de Cananéia, e Fernandez et al. (2014) encontraram concentrações de $\mathrm{Zn}$ e $\mathrm{Cu}$ acima dos níveis permitidos de $30 \mathrm{mg} \mathrm{kg}$, em fígados do peixe Mugil curema de toda área estuarina (ANVISA, 1998). GussoChoueri et al. (2018), analisando as concentrações de $\mathrm{Pb}$ no músculo de peixes da espécie (Cathorops spixii) de toda a região estuarina, verificaram valores de até $14,64 \mathrm{mg} \mathrm{kg}^{-1}$, muito acima dos de $0,3 \mathrm{mg} \mathrm{kg}^{-1}$ permitidos pela legislação brasileira (ANVISA, 1998). Os autores ainda ressaltaram que o consumo acentuado destes peixes pela população local pode trazer riscos à saúde humana (GUSSO-CHOUERI et al., 2018).

\section{CONCLUSÃO}

A análise da concentração dos sulfetos volatizados por acidificação (SVA) e biodisponibilidade de íons metálicos extraídos simultaneamente (SEM) se mostrou uma ferramenta eficaz para se averiguar a biodisponibilidade e toxicidade de metais nos sedimentos estuarinos do CELIC. Os resultados da avaliação das variações espaciais e temporais dos metais $\mathrm{Fe}, \mathrm{Zn}, \mathrm{Mn}, \mathrm{Co}, \mathrm{Cu}, \mathrm{Cr}, \mathrm{Cd}, \mathrm{Pb}$ e $\mathrm{Ni}$ e a sua biodisponibilidade nestes sedimentos juntamente com a determinação de nutrientes permitiram determinar os pontos de maior impacto antrópico dentro do sistema.

A média dos metais fortemente biodisponíveis na região deu-se na ordem de $\mathrm{Fe}>\mathrm{Mn}>\mathrm{Zn}>\mathrm{Pb}>\mathrm{Cu}$, com os pontos localizados a Norte (N1, N2 e N3), 
apresentando as maiores concentrações de metais. No entanto, todos os pontos apresentaram razão SEM/SVA $>1$ para os íons metálicos $\mathrm{Pb}, \mathrm{Cd}$, Ni, $\mathrm{Cu}$ e Zn, em ao menos uma das coletas, com maior toxicidade observada para os sedimentos nas proximidades de Iguape. Estes resultados indicam o potencial destes sedimentos de causar efeitos adversos a biota em ambas as regióes. A toxicidade vista provavelmente se dá pelos baixos valores de SVA observados nas camadas superficiais dos sedimentos com relação às altas quantias de metais bivalentes encontradas.

Para a região de Cananéia (Sul), os resultados sugerem que apesar de se observar uma menor atividade antrópica nesta área, os sedimentos dos pontos analisados estão sujeitos a contaminações pelo lançamento direto de esgotos domésticos, a deposição incorreta de lixo e a presença de embarcações. Nesta área os teores de fósforo nos sedimentos podem ser atribuídos tanto a contribuições naturais de fontes terrígenas, mas também a contribuições em maior ou menor grau do centro urbano da região. Para os metais, os valores de chumbo excederam os limites da legislação brasileira de nível I nos pontos $\mathrm{S2}$, S3 e S5 e de zinco nos pontos S1 a 54 em ao menos uma das coletas.

Na regiãode Iguape (Norte) odesague do Rio Ribeira de Iguape garante grande aporte de fósforo a área, proveniente de fontes terrígenas, de atividades agrícolas e mineradoras e dos efluentes domésticos do centro urbano de Iguape. A entrada das águas deste rio garante ainda a elevada presença de metais nestes sedimentos, onde todos os pontos analisados a Norte (N1 a N3) tiveram concentrações de Pb, Cu e Zn acima das permitidas pela legislação nacional em ambas as coletas, com valores superiores aos que se prevêem efeitos adversos à biota. A correlação encontrada entre estes três metais $(\mathrm{Pb}, \mathrm{Cu}$ e $\mathrm{Zn})$ suporta a ideia de que sejam provenientes da mesma fonte, ou seja, das antigas atividades mineradoras que se deram nas margens do Rio Ribeira de Iguape.

Deste modo conclui-se que efeitos deletérios deixados pelas antigas atividades de mineração na bacia do Rio Ribeira de Iguape ainda estão presentes dentro do ambiente aquático do CELIC. Assim, a quantificação e o estudo do comportamento de metais e seus possíveis efeitos adversos na região continuam tendo grande importância para o monitoramento contínuo da qualidade deste ecossistema e do grau de exposição da biota local a estes contaminantes. 


\section{AGRADECIMENTOS}

Ao CNPq; à Fundação Araucária; à CAPES; à UTFPR e ao FINEP (CT-Infra/2010 e CT-Infra/2011 pelos Recursos FNDCT, subprojeto NIPTA).

\section{REFERÊNCIAS}

ABESSA, D. M. S.; MORAIS, L. G.; PERINA, F. C.; DAVANSO, M. B.; BURUAEM, L. M.; MARTINS, L. M. P.; SÍGOLO, J. B.; RODRIGUES, V. G. S. Toxicidade de águas e sedimentos em um rio afetado por atividades mineradoras pretéritas. $\mathrm{O}$ Mundo da Saúde, v. 36, n. 4, p. 610-618, 2012.

ABESSA, D. M. S.; MORAIS, L. G.; PERINA, F. C.; DAVANSO, M. B.; RODRIGUES, V. G. S.; MARTINS, L. M. P.; SÍGOLO, J. B. Sediment geochemistry and climatic influences in a river influenced by former mining activities: The Case of Ribeira de Iguape River, SP-PR, Brazil. Open Journal of Water Pollution and Treatment, v. 1, n. 1, p. 43-53, 2014.

ALBA, J. M. F.; SOUZA FILHO, C. R.; FIGUEIREDO, B. R. Análise da assinatura geoquímica de solos e de sedimentos de corrente no Vale do Ribeira (SP) por meio de um sistema de informação geográfica. Revista Brasileira de Geociências, v. 38, n. 1, p. 66-77, 2008.

AMORIM, E. P.; FÁVARO, D. I. T.; BERBEL, G. B. B.; BRAGA, E. S. Assessment of metal and trace element concentrations in the Cananéia estuary, Brazil, by neutron activation and atomic absorption techniques. Journal of Radioanalytical and Nuclear Chemistry, v. 278, n. 2, p. 485-489, 2008.

AMORIM, E. P.; FÁVARO, D. I. T.; BRAGA, E. S. Toxic Metal Assessment in estuarine sediments from Cananéia, São Paulo, Brazil, determined by Atomic Absorption Spectrometry. A expressão In: XIII CONGRESSO BRASILEIRO DE GEOQUÍMICA, 2011, Gramado, Rio Grande do Sul.

ANDERSEN, J. M. An ignition method for determination of total phosphorus in lake sediments. Water Research, v. 10, n. 4, p. 329-331, 1976. 
ANVISA - Agência Nacional de Vigilância Sanitária. Portaria ANVISA nº 685, de 27 de agosto de 1998, Brasília, 1998.

APHA - American Public Health Association. Standard Methods for the examination of water and wasterwater. $21^{\mathrm{a}}$ ed. Washington, 2005.

ARMELIN, J. A.; SAIKI, M. INAA applied to the multielemental characterization of a sedimentary column: a contribution to oceanographic studies. Journal of Radioanalytical and Nuclear Chemistry, v. 282, p. 91-94, 2009.

BARBIERI, E.; MARQUEZ, H. L. A.; CAMPOLIM, M. B.; SALVARINI, P. I. Avaliação dos impactos ambientais e socioeconômicos da aquicultura na região estuarina-lagunar de Cananéia, São Paulo, Brasil. Rev. Gestão Costeira Integrada, v. 14, n. 3, p. 385-398, 2014.

BARCELLOS, R. L.; BERBEL, G. B. B.; BRAGA, E. S.; FURTADO, V. V. Distribuição e características do fósforo sedimentar no sistema estuarino lagunar de Cananéia-Iguape, estado de São Paulo, Brasil. Geochimica Brasil, v. 19, n. 1, p. 22-36, 2005.

BORAN, M.; ALTINOK, I. A Review of Heavy Metals in Water, Sediment and Living Organisms in the Black Sea. Turkish Journal of Fisheries and Aquatic Sciences, v. 10, p. 565-572, 2010.

BRICHTA, M.; GAETA, S. A. Biomassa e produção autotrófica planctônica no complexo estuarino-lagunar Iguape-Cananéia, São Paulo. Tese (Doutorado). Instituto Oceanográfico - USP, São Paulo, 2000.

CAMPANA, O.; RODRÍGUEZ, A.; BLASCO, J. Heavy metals bio-availability in the Guadalete River Estuary (SW Spain). Ciências Marinas, v. 31, p. 135-147, 2004.

CASTRO, F. J. V. Disponibilidade dos metais Cd e Pb e do metalóide As na bacia hidrográfica do rio Ribeira de Iguape e afluentes: Uma avaliação da contaminação ambiental, 2012. Dissertação (Mestrado em Ciências). Universidade de São Paulo, São Paulo, 2012.

CONAMA (Conselho Nacional do Meio Ambiente). Resolução CONAMA n ${ }^{\circ} 344$, de 25 de março de 2005. Brasília, 2004. 
COTTA, J. A. O.; REZENDE, M. O. O.; PIOVANI, M. R. Avaliação do teor de metais em sedimento do Rio Betari no Parque Estadual Turístico do Alto Ribeira - PETAR, São Paulo, Brasil. Química Nova, v. 29, n. 1, p. 40-45, 2006.

CUNHA, F. G.; FIGUEIREDO, B. R.; PAOLIELLO, M. M. B.; DE CAPITANI, E. M. SAKUMA, A. M. Human and environmental lead contamination in the Upper Ribeira Valley, southeastern Brazil. Terrae, v. 2, p. 28-36, 2005.

DI TORO, D. M.; MAHONY, J. D.; HANSEN, D. J.; SCOTT, K. J.; CARLSON, A. R.; ANKLEY, G. T. Acid volatile sulfide predicts the acute toxicity of cadmium and nickel in sediments. Environmental Science \& Technology, v. 26, n. 1, p. 96-101, 1992.

FERNANDEZ, W. S.; DIAS, J. F.; BOUFLEUR, L. A.; AMARAL, L.; YONEAMA, M. L.; DIAS, J. F. Bioacumulation of trace elements in hepatic and renal tissues of the white mullet Mugil curema Valenciennes, 1836 (Actinopterygii, Mugilidae) in two coastal systems in southeastern Brazil. Nuclear Instruments and Methods in Physics Research B, v. 318, p. 94-98, 2014.

GARCIA, K. S.; OLIVEIRA, O. M. C.; MADDOCK, J. E. L.; SOARES, S. A. R.; CELINO, J. J. Variação espacial de sulfetos voláteis acidificados e biodisponibilidade de metais extraídos simultaneamente em sedimentos ao norte da Baía de Todos os Santos, Bahia. Cadernos de Geociências, v. 11, n. 1-2, p. 36- 48, 2014.

GUIMARÃES, V.; SÍGOLO, J. B. Detecção de contaminantes em espécie bioindicadora (Corbicula fluminea) - Rio Ribeira de Iguape - SP. Química Nova, v. 31, n. 7, p. 1696-1698, 2008a.

GUIMARÃES, V.; SÍGOLO, J. B. Interação de resíduos da metalurgia com sedimentos em suspensão - Rio Ribeira de Iguape. Revista Geologia USP, v. 8, n. 2, p. $1-10,2008 b$.

GUSSO-CHOUERI, P. K.; ARAÚJO, G. S.; CRUZ, A. C. F.; STREMEL, T. R. O.; CAMPOS, S. X.; ABESSA, D. M. S.; RIBEIRO, C. A. O.; CHOUERRI, R. B. Metals and arsenic in fish from a Ramsar site under past and present human pressures: Consumption risk factors to the local population. Science of the Total Environment, 628-629, p. 621-630, 2018. 
HOOP, M. A. G. T.; HOLLANDER, H. A.; KERDIJK, H. N. Spatial and seasonal variations of acid volatile sulphide (AVS) and simultaneously extracted metals (SEM) in Dutch marine and freshwater sediments. Chemosphere, v. 35, n. 10, p. 2307-2316, 1997.

MACHADO, I. C.; MAIO, F. D.; KIRA, C. S.; CARVALHO, M. F. H. Estudo da Ocorrência dos metais pesados $\mathrm{Pb}, \mathrm{Cd}, \mathrm{Hg}$, Cu e $\mathrm{Zn}$ na ostra de mangue Crassostrea brasiliana do estuário de Cananéia - SP, Brasil. Revista Instituto Adolfo Lutz, v. 61, n. 1, p. 13-18, 2002.

MACHADO, A. A. S.; SPENCER, K.; KLOAS, W. TOFFOLON, M.; ZARF, L. Metal fate and effects in estuaries: A review and conceptual model for better understanding of toxicity. Science of The Total Environment, v. 541, p. 268- 281, 2016.

MAHIQUES, M. M. et al. Anthropogenic influences in a lagoonal environment: a multiproxy approach at the Valo Grande mouth, Cananeia-Iguape system (SP Brazil). Brazilian Journal of Oceanography, v. 57, n. 4, p. 325-337, 2009.

MAHIQUES, M. M.; FIGUEIRA, R. C. L.; SALAROLI, A. B.; ALVES, D. P. V.; ALVES, C. G. 150 years of anthropogenic metal input in a Biosphere Reserve: the case study of the Cananéia-Iguape coastal system, Southeastern Brazil. Environmental Earth Sciences, v. 68, n. 4, p. 1073-1087. 2013.

MALUF, J. C. C. Estudo dos metais traço (zinco, cádmio e chumbo) em duas regióes do Complexo Estuarino-Lagunar de Cananéia-Iguape (SP) sob diferentes pressões antrópicas, 2009. 145 f. Dissertação (Mestrado em Oceanografia), Instituto Oceanográfico da Universidade de São Paulo, 2009.

MORAIS, L. G.; ABESSA, D. M. S. PSR framework applied to the coastal management of "Complexo Estuarino-Lagunar Iguape-Cananéia" - CELIC (São Paulo, Brazil), in terms of sanitation and public health. Revista de Gestão Costeira Integrada, v. 14, n. 4, p. 625-635. 2014.

OLIVEIRA, J. L.; EUGÊNIO, A.; AZEVEDO, J. C. R.; NOZAKI, J. Influence of metal oxides in the colorimetric determination ofacid volatile sulfide in sediments. Acta bydrochim. Hydrobiol., v. 34, 383-388, 2006. 
OLIVEIRA, R. C. B.; MARINS, R. V. Dinâmica de metais-traço em solo e ambiente sedimentar estuarino como um fator determinante no aporte desses contaminantes para o ambiente aquático. Revista Virtual Química, v. 3, n. 2, p. 88-102, 2011.

POMPÊO, M.; PADIAL, P. R.; MARIANI, C. F.; SILVA, S. C.; CARLOS, V. M.; SILVA, D. C. V. R.; PAIVA, T. C. B.; BRANDIMARTE, A. L. Biodisponibilidade de metais no sedimento de um reservatório tropical urbano (reservatório Guarapiranga-São Paulo (SP), Brasil): há toxicidade potencial e heterogeneidade espacial? Geochimica Brasiliensis, v. 27, n. 2, p. 104-119, 2013.

RODRIGUES, V. G. S.; FUJIKAWA, A.; ABESSA, D. M. S.; HORTELLANI, M. A.; SARKIS, J. E. S.; SÍGOLO, J. B. Uso do bivalve límnico Anodontites tenebricosus (Lea, 1834) no biomonitoramento de metais do Rio Ribeira de Iguape. Química Nova, v. 35, n. 3, p. 454-459, 2012.

SAITO, R. T.; FIGUEIRA, R. C. L.; TESSLER, M. G.; CUNHA, I. I. L. 210Pb and 137Cs geochronologies in the Cananeia-Iguape Estuary (São Paulo, Brazil). Journal of Radioanalytical and Nuclear Chemistry, v. 249, n. 1, p. 257-261, 2001.

SAITO, R. T.; CUNHA, I. I. L.; FIGUEIRA, R. C. L.; TESSLER, M. G. 210Pb and 210Po levels in sediments, water, and bioindicators in the Cananeia-Iguape estuary - Sao Paulo - Brazil. Czechoslovak Journal of Physics, n. 53, n. 1, p. 75-81. 2003.

SILVEIRA, P. B.; ALVES, P. S.; ALMEIDA, A. M.; SILVA FILHO, C. A.; VALENTIM, E.; HAZIN, C. A. Avaliação da biodisponibilidade de metais em sedimentos de manguezais da área do Complexo Estuarino de Suape-PE. Scientia Plena, v. 9, n. 8, p. 1-8, 2013.

TESSLER, M. G.; SOUZA, L. A. P. Dinâmica sedimentar e feições sedimentares identificadas na superfície de fundo do Sistema Cananéia-Iguape, SP. Revista Brasileira de Oceanografia, v. 46, n. 1, p. 69-83, 1998.

Recebido em: 09/11/2017

Aceito em: 06/06/2018 